\title{
Diagnostische Notizen
}

Untersuehunge $\prod$ über Glaukom und Blutdruck.

Dr. R. Kümmell, Assistent der Universitäts-Augenklinik Erlangen, hat, ausgehend von

Untersuchungen an exquisit hämorrhagischen Glau-komen, bei denen ein Zusammenhang mit einer allgemeinen Erkrankung des Gefäßsystems bezw. Nierenleiden naheliegend und erweisbar war, auch die ge $\Lambda$ vöhnlichen G laukomformen in den Kreis seiner Untersuchungen gezogen.

Diagnostisch $\beta$ N"otizen.

181

Das Thema ist neuerdings auch von anderer Seite [Kramer]1) u. A.) bearbeitet.

Kümmell kommt - nicht immer ganz iibereinstimmend mit Kramer - zu folgenden Resultaten (auf Grund eines allerdings nu $\mathrm{\Gamma}$ kleinen Untersuchungsmaterials von 30 Glaukomatösen und entsprechen-der Kontrollfälle):

Bei alien diesen Glaukomkranken fand sich gegenüber gleichartigen Gesunden eine wesentliche Steigerung des Durchschnittswertes des Blutdracks2).

Ferner finden sich meist noch andere Symptome für eine Erkran-kung des cardio-vaskulären und renalen Systems, so daß man das Glaukom überhaupt in den meisten Fallen als einen Teil der Allgemein-erkrankung ansehen muß.

Einschränkend führt Verf. hierzu aus:

Diese Norm gilt nicht ohne Ausnahmen.

Es kommen Glaukome ohne Blutdruckerhöhung vor. Man kann aber wohl sagen: Ein solches Glaukom muß den Verdacht auf ein atypi-sches Verhalten erwecken (in einem beobachteten Falle ohne Blutdruckerhöhung war eine intraokulare Tumormetastase Ursache der klinischen Zeichen eines akut entzündlichen Glaukoms).

Umgekehrt gibt es hohen Blutdruck ohne Glaukom.

Denn der hohe Blutdruck ist nur ein Moment unter denUrsachen des Glaukoms.

Damit Glaukom entstehen kann, müssen noch andere Momente wirksam sein, die wir noch nicht alle kennen.

$\mathrm{Zu}$ denken ist an lokale okulare Arteriosklerose. Man kann ihr in Gemeinschaft mit gleichzeitig vorhandenem erhöhtem Blutdruck wohl einen größeren Raum in der Pathogenese einräumen, ohne die relativ geringe Zahl von Glaukomkranken für auffällig zu halten. Kommen doch auch z. B. Netzhautblutungen infolge Arteriosklerose im Vergleich zu den Allgemeinerkrankungen der Gefäße relativ selten vor.

Von Bedeutung erscheinen die Ergebnisse nach K., abgesehen von dem allgemeinen wissenschaftlichen Interesse, nach zwei Richtungen hin:

Dem Verschluß des Kammerwinkels kommt nur eine sekundäre Bedeutung zu.

Neben der anerkannten Glaukomtherapie kommt für einzelne Fälle Herabsetzung des Blutdrucks durch Aderlässe in Erage (nach dem Vor-gang von Webster Fox).

Die Schl·ußfolgerungen werden im einzelnen begründet.

(v. Gräfes Arch. f. Ophthalm. 1911. Bd. LXXIX. S. 183.)

Junius. 
M v. Gräfes Arch. f. Ophthalm. 1910. Bd. LXXIII.

2. 2) Festgestellt mit besonderer Messungsmethode. Die Unterschied $\theta$ sind am größten beim systolischen Blutdruck

geringer beim diastolischen

noch geringer bei der Druckamplitude

so daß der Hauptwert auf die Be-stimmung des systolischen Drackes zu legen ist. 